\title{
Implementation of User-Pairing Algorithm by using Heuristic Technique for OFDMA
}

\author{
Manpreet Singh ${ }^{1}$, Preeti Gulati ${ }^{2}$ \\ M.Tech Student, ECE Department, CGC Landran, Mohali, India ${ }^{1}$ \\ Assistant Professor, ECE Department, CGC Landran, Mohali, India ${ }^{2}$
}

\begin{abstract}
In this term paper; the problem of increasing efficiency in OFDMA had been abridged into an utmost biased similar dilemma which can have polynomial time solution or it can be further optimized to get better results. In this research work, pairing algorithm performance is improved by the using, Heuristic Algorithm technique which is based on strength of signal. The potency of signal is calculated and then couple the users based on their signal potency. There is no need to locate the distance of the users. Simulation results specify that our heuristic algorithm attain asymptotic performance of optimal allocation algorithm with much lower complexity. Though we just consider some classes of services in the simulation, it is easy to expand more modules of services without escalating intricacy. The outcome of the best prejudiced identical algorithm had been evaluated and will be compared with other method to show its complexity. They had also used matching algorithms to find the location information of the users.
\end{abstract}

Keywords: OFDMA, Heuristic Pairing Algorithm, FFT, SNR and BER

\section{INTRODUCTION}

However, Heuristic pairing Algorithm is extensively computationally less expensive than optimal pairing as it is exercises straightforward assessment operations to explore for the greatest load in a solo iteration. Moreover it is less complex as it compares only SNR as compared to the distance of the user.

Heuristic Pairing Algorithm approaches two types of pairing.

I Max-Max Pairing

II Max-Min Pairing

Frequency Division Multiplexing (FDM) has been employ for an extensive time to take more than single signal over a telephone line. FDM splits the channel bandwidth into sub-channels and broadcast several fairly low rate signals by transporting each signal on a separate carrier frequency. To make sure that the signal of single sub-channel did not moderately cover with the signal from a contiguous one, few guard-band was absent between the dissimilar subchannels. Obviously, this guard-band led to inefficiencies.

In order to resolve the bandwidth effectiveness difficulty, orthogonal frequency division multiplexing was projected, where the dissimilar carriers are orthogonal to one another. By means of OFDM, it is likely to have partly cover subchannels in the frequency domain, thus escalating the transmission rate. This carrier spacing recommends optimal spectral effectiveness. Nowadays, OFDM has matured to be the mainly trendy communication system in high-speed communications. OFDM is becoming the elected modulation method for wireless communications. OFDM can offer large data rates with enough strength to radio channel destruction.

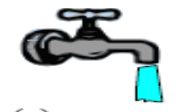

(a)

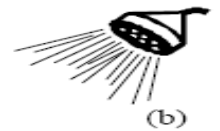

Figure 1(a). FDM single carrier

(b).Orthogonal-FDM

\section{ALGORITHM}

In Heuristic Paring algorithm, Pairing is done between the users on the base of signal strength. The signal strength is calculated based on the SNR. Users which will be higher in signal strength will make pair with others users, so that they can help each other in cooperative network.

The following are the heuristic pairing algorithm

I. Max-max pairing.

This algorithm couples users with the goal of forthcoming the most favourable ability at a significantly abridged computational intricacy. A credence matrix $\mathrm{W}$ with zeros on core oblique and symmetric entries $m[W]_{i j}=[W]_{j i}=$ $C_{i}, D+C_{j}, D$ is recognized, where $i$ and $j$ are impending pairs. The algorithm is officially obtainable as follows: Initialize an vacant coupling $P$. Choose the biggest aspect from $\mathrm{W}$, for example $[\mathrm{W}] i j$, and structure the pair by supplement $P$ with $i$ and $j$. Revise $\mathrm{W}$ by eradicate the rows and columns equivalent to the pair created. Continue until $P$ is absolute and each and every one node has been matched. This algorithm has $O\left(N^{3}\right)$ time intricacy and consequently react likewise to the alter in the number of contribution (i.e., users to be matched) like the optimal algorithm. Though, max-max pairing is appreciably computationally less dear than finest pairing as it is uses straightforward assessment actions to look for the utmost load in a particular monotony. It is furthermore replicate by the simulation times.

\section{Max-min pairing}

This heuristic algorithm is planned to tackle the structure outage likelihood. We set up with the feeblest user (in provisos of the SNR to the goal) in the cell and couple it by the user contain the sturdy of the feeble of the sourcerelay and relay-destination relations, since the outage performance is forever dogged by the weaker of the two links and carry on for other users. Tis algorithm had a point difficulty of $O\left(N^{2}\right)$ and is officially accessible as pursue: 
Initialize an blank pairing $P$. Opt for a node $i$ with the feeblest $\gamma i, D$ and match up it with node $j$ with $\left.\max \left[\min \gamma_{\mathrm{ij}}, \gamma_{\mathrm{j}}, \mathrm{D}\right)\right]$. Supplement the pairing $P$ with the pair created in and revise the set of entitled nodes. Carry on from until $P$ is full and every single one node has been matched. Max-min matching is calculation capable as it is based on straightforward evaluation actions; it is also reveal in the common simulation period.

\section{Heuristic algorithm consists of five steps:}

(a) Pre estimates initially the number of subcarriers to be allocated to each user.

(b) Choose antenna and subcarriers for every user based on the conventional number of subcarriers.

(c) Pre estimates the number of bits allocation to every subcarrier and alter the number of bits agreement to the necessity of the user's minimum transmission rate and minimum broadcast power; then fill bits for each subcarrier.

(d) Allocate lasting subcarriers to the user who's Quality of service obligation is not fulfilled.

(e) Allocate power to each subcarrier according to Quality of Service.
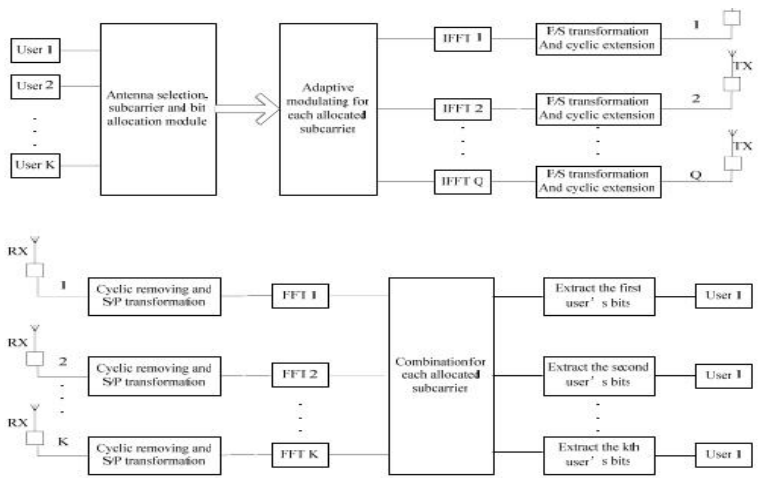

Figure 2. Functioning of Heuristic algorithm

\section{RESULT AND DISCUSSION}

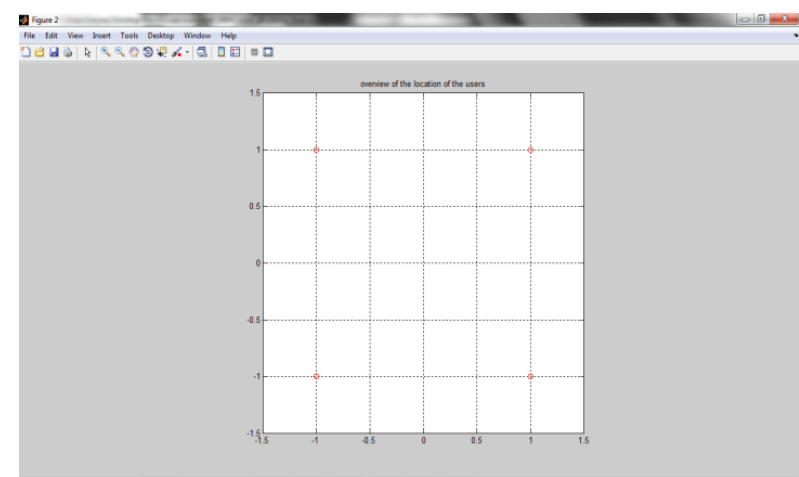

Figure 3. Overview of the location of the users

In the OFDM system execution Fast Fourier Transform (FFT) and its opposite (IFFT) were used to execute the modulation and demodulation process. The Fourier transform permit us to divide actions in time domain to actions in frequency domain. OFDM system employs discrete Fourier transform (DFT) to modulate and demodulate matching information.

We had also used similar algorithms to locate the position information of the users.

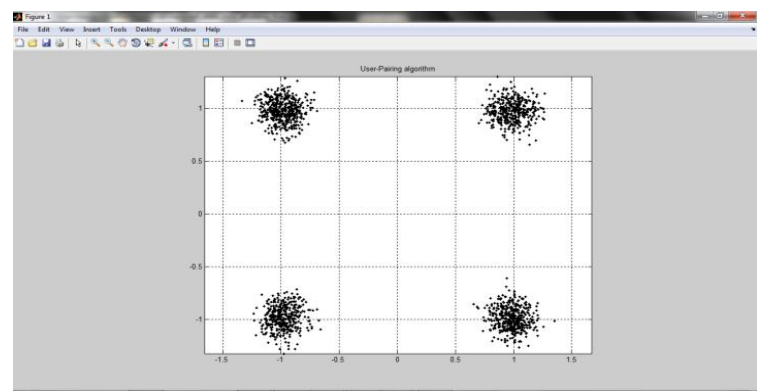

Figure 4. Exact location of the users by using location finding algorithm

In the OFDM system implementation, real-valued matching parameters and their opposite are used to execute the modulation and demodulation actions.

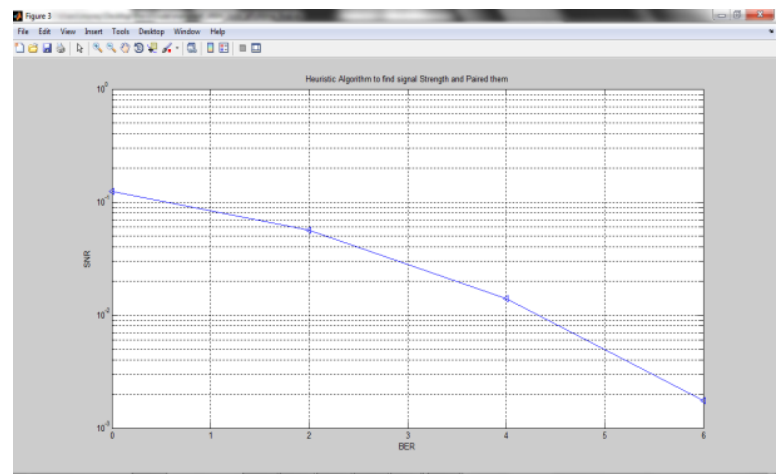

Figure 5. Comparison between SNR and BER

Heuristic algorithm technique which is based on strength of signal rather than distances that was done in previous work. So we used this algorithm to accumulate the time, intricacy and making it less expensive than the previous one. In the figure shown graph between SNR and BER. This contains the potency of the signal and paired them.

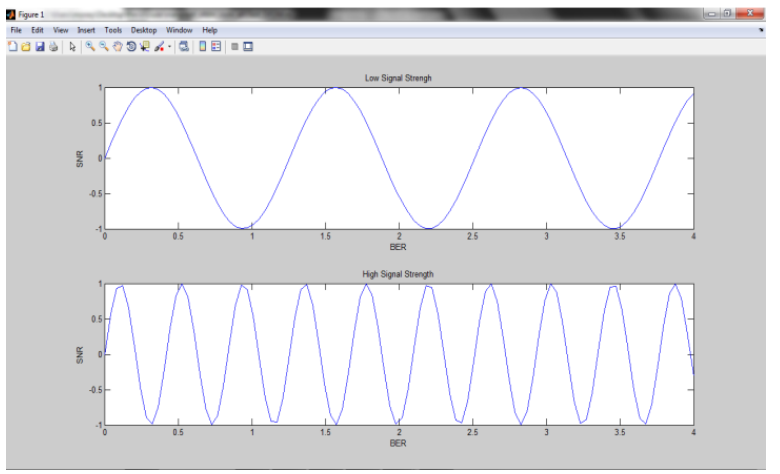

Figure 6. Comparison between strength of the signal

The whole simulation process to be carried out is alike to that of OFDM with similar scheme. For fastidious SNR value system is simulated and parallel probability of error (Bit Error Rate, BER) is calculated. As we go on escalating the SNR value, bit error rate lessens.

\section{IV.CONCLUSION}

This paper includes that we used Heuristic algorithm technique which is based on strength of signal rather than distance that was done in previous work. So we used this algorithm to accumulate the time, intricacy and making it less expensive than the previous one. The OFDM makes well-organized exercise of obtainable range by permitting 
partly covering among the carriers. It principally switches the elevated data rate stream in to numerous matching inferior data rate streams and thus eradicating the frequency discriminatory pairing there is no need to locate the distance of the users. So it will not only make error, but also make the system less complex and less expensive. Simulation results specify that our heuristic algorithm attain asymptotic performance of optimal allocation algorithm with much lower complexity. Though we just consider some classes of services in the simulation, it is easy to expand more modules of services without escalating intricacy. With MATLAB software, the presentation of OFDM system was experienced for digital modulation techniques namely pairing and matching. The performance of OFDM system was also experienced for two different types of users, and by using Heuristic Algorithm the performance of the users weathered, based on signal to Noise Ratio and paired them accordingly.

\section{ACKNOWLEDEMENT}

At this phase of work, as the rather prolonged work brings to close, I am becoming increasingly present to the detail that exploration can certainly be an pleasant and satisfying skill, even though the boredom and hard work concerned. This paper is strictly the finale of the support, inspiration, liberal help and wisdom of my supervisor, Mrs.Preeti Gulati, Assistant Professor, CGC-College of Engineering. I am highly grateful to Dr.Rinkesh Mittal, Head, Department of Electronics \& Communication Engineering, CGC-COE, Landran, for providing this chance to carry out the current work. I am also thankful to all the staff members of the ECE department for their full co-operation and help. Mrs.Preeti Gulati's sophisticated exploration recommendation in the extremely primary phase never leave in lurch in probe during the long era of legwork. I could find out the method of systematizing and writing superiority research stuff only because of his scholarly teachings during the work.

\section{REFERENCES}

[1] A. Sendonaris, B. Aazhang and E. Erkip. "User Cooperation Diversity- Part I: System Description." IEEE Trans. Commun., 51(11):1927-1938, Nov. (2003).

[2] A. Mukherjee, H.M. Kwon "General Auction-Theoretic Strategies for Distributed Partner Selection in Cooperative Wireless Networks." IEEE Trans. Communications, 58(10):2903-2915, October (2010)

[3] Chow, Sheng-Mou Yu and Yinhua Wang, QPSK Modulation and Demodulation, "ELE 791 Software Radio Design, Michael", , Syracuse University, Dec $14^{\text {th }}$ (2004)

[4] C. Ng and C. Sung. "Low Complexity Subcarrier and Power Allocationfor Utility Maximization in Uplink OFDMA Systems." IEEE Trans. Wireless Commun., 7(5): 1667-1675, May (2008)

[5] Hua Mu, Jianwei Meixia, Tao Huang and Wenbing Dang, and, "Subcarrier-Pair Based Resource Allocation for Cooperative MultiRelay OFDM Systems" IEEE TRANSACTIONS ON WIRELESS COMMUNICATIONS, VOL. 9, NO. 5, MAY (2010)

[6] J.W. Mark, L. Cai, V. Mahinthan, and X. Shen "Partner Selection Based on Optimal Power Allocation in Cooperative-Diversity Systems." IEEE Trans. Vehicular Tech., 57(1): 511-520, Jan. (2008)

[7] K. Kim, S.-L. Kim and Y. Han and "Joint Subcarrier and Power Allocation in Uplink OFDMA Systems." IEEE Commun. Lett., 9(6): 526-528, Jun. (2005)
[8] L. Gao and S. Cui. "Efficient Subcarrier, Power and Rate Allocation with Fairness Consideration for OFDMA Uplink." IEEE Trans. Wireless Commun., 7(5): 1507-1511, May (2008)

[9] O. Kaya and S. Bakım. "Cooperative Strategies and Achievable Rates for Two User OFDMA Channels." IEEE Trans. Wireless Commun., 10(12): 4029-4034, Dec. (2011)

[10] O. Kaya and S. Bakım. "Optimum Power Control for Transmitter Cooperation in OFDMA Based Wireless Networks." In Proc. IEEE Globecom, Multicell Cooperation Workshop, Houston, TX, Dec. (2011)

[11] S. Kim, W. Shim and Y. Han. "Fairness-Aware Resource Allocation in a Cooperative OFDMA Uplink System." IEEE Trans. Veh. Technol., 59(2): 932-939, Feb. (2010)

[12] S. Baksi, O. Kaya, T. Biyikoglu, Optimal and Near-optimal Partner Selection Algorithms in Cooperative OFDMA, IEEE Wireless Communications and Networking Conference (WCNC 2012), Paris, France, April (2012) 